Research Article

\title{
Interaction of Self-Sustained Pulses in Tunnel-Diode Oscillator Lattices
}

\author{
Koichi Narahara \\ Department of Electrical and Electronic Engineering, Kanagawa Institute of Technology, 1030 Shimoogino, Atsugi, \\ Kanagawa 243-0292, Japan \\ Correspondence should be addressed to Koichi Narahara; narahara@ele.kanagawa-it.ac.jp
}

Received 1 October 2021; Accepted 18 November 2021; Published 15 December 2021

Academic Editor: Alessandro Lo Schiavo

Copyright (c) 2021 Koichi Narahara. This is an open access article distributed under the Creative Commons Attribution License, which permits unrestricted use, distribution, and reproduction in any medium, provided the original work is properly cited.

A one-dimensional lattice in tunnel-diode (TD) oscillators supports self-sustained solitary pulses resulting from the balance between gain and attenuation. By applying the reduction theory to the device's model equation, it is found that two relatively distant pulses moving in the lattice are mutually affected by a repulsive interaction. This property can be efficiently utilized in equalizing pulse positions to achieve jitter elimination. In particular, when two pulses rotate in a small, closed lattice, they separate evenly at the asymptotic limit. As a result, the lattice loop can provide an efficient platform to obtain low-phase-noise multiphase oscillatory signals. In this work, the interaction between two self-sustained pulses in a TD-oscillator lattice is examined, and the properties of interpulse interaction are validated by conducting several measurements using a test breadboarded lattice.

\section{Introduction}

In this paper, the dynamics of interacting self-sustained pulses developed in a one-dimensional lattice in tunneldiode (TD) oscillators are characterized. For this purpose, an oscillator, which is driven by a single DC voltage $v_{B}$ that biases the TD, is employed. As a result, the TD exhibits gain by its own negative differential resistance. Using bifurcational and experimental characterization, it is found that a self-sustained pulse in a TD-oscillator lattice has a rather long tail, and its velocity significantly depends on $v_{B}[1]$. As a result, the pulse propagates faster and becomes larger as $v_{B}$ increases. Furthermore, the pulse tail exhibits a small oscillation if the inductors' parasitic resistances are negligible. In contrast, the pulse oscillatory tail becomes exponentially damped for finite parasitic resistances. The measured solitary pulse is allowed to develop for a restricted range of $v_{B}$ values around the TD's peak voltage. Below the lower bound of this range, the input pulse is monotonically attenuated and finally disappears. In contrast, above the upper bound of this range, the lattice exhibits synchronous oscillation, and the solitary pulse is replaced by phase waves.
The long tail of the solitary pulse can affect the propagation properties of the subsequent pulse because the pulse velocity significantly depends on the baseline voltage. To examine the type of interaction between two successive pulses, the reduction theory [2] is applied to the governing equations of a TD-oscillator lattice. It is found that, for no parasitic resistances, this interaction becomes either repulsive or attractive, depending on the separation distance between the pulses. However, for finite parasitic resistances, the separation distance tends to approach a specific value. Similar wave interaction has been observed in liquid film coating a vertical fiber $[3,4]$, e.g., where a drop-like wave interacts with a nearby one. The existence of bound states has been clearly described using the generalized Kuramoto-Sivashinsky equation [5].

The interacting self-sustained pulses can be utilized in several signal processing applications. Let us consider an infinite pulse train inserted into a straight lattice. When a repulsive interaction dominates the pulse dynamics, the separation between any two adjacent pulses is asymptotically equalized. In this case, the TD lattice can reduce the timing jitter, which is initially present in the pulse train. In addition, two rotary self-sustained pulses move in the lattice loop in 
such a way that one occupies the other's antipodal point. This can be achieved by setting half of the loop size to be less than the asymptotically converging separation value. This mechanism is expected to be applied to phase-noise reduction. In this study, the pulse properties in the lattice are examined using TDs. Furthermore, validation was carried out with flexible low-frequency experiments. However, once resonant-tunneling diodes (RTDs) [6-9] are implemented in place of TDs, the device potentially achieves higher operation frequency than CMOS circuits.

In Section 2, the dynamics of relatively distant interacting nonlinear pulses are characterized in the reduction theory framework. A reduced model representing the interaction of self-sustained pulses in a TD-oscillator lattice is initially developed, after describing the fundamental properties of the lattice. The experimental results obtained using a test breadboarded lattice are presented in Section 3. In Section 4 , the pulse dynamics in a lattice loop are predicted using the reduced model, and the equalization of interpulse spacing is achieved using the 4th-order Runge-Kutta calculation.

\section{Reduced Description of the Pulse Interaction}

The unit-cell structure of the lattice under investigation is shown in Figure 1. The lossy inductor represented by $L_{s h}$ and $R_{s h}$ is connected in parallel with a capacitance $C$. The nonlinear current source $I_{D}$ represents the TD. The $L_{s h}$ and $C$ pair establishes resonance resulting in voltage oscillation. By the application of the DC bias voltage $v_{B}$ the TD's negative differential resistance compensates for the oscillation damping caused by $R_{s h}$. We consider the dynamics of self-sustained solitary waves developed in an inductively coupled system of unit-cell oscillators. The coupling is established by the lossy inductor represented by $L_{s e}$ and $R_{s e}$. In this work, the $L_{s e}$ and $L_{s h}$ values are commonly set as $L_{s e}=L_{s h} \equiv L$ (correspondingly, $R_{s e}=R_{s h} \equiv R$ ). The resulting one-dimensional lattice supports a self-sustained pulse when the $v_{B}$ value is set in the restricted range around the TD's peak voltage [1]. The near end of the lattice is connected to a pulse source to excite the self-sustained solitary pulses and the far end is connected to a load.

The continuous spatial variable $x$ is introduced instead of the cell address. The number of cells included in the unit length is defined as $N_{c}$. Then, in the long-wavelength approximation, the governing equations of the solitary pulses in the lattice are given as follows:

$$
\begin{aligned}
N_{c} L \frac{\partial i}{\partial t}+N_{c} R i & =-\frac{\partial v}{\partial x}, \\
N_{c} C \frac{\partial v}{\partial t}+N_{c} I_{D}(v)+i_{s h} & =-\frac{\partial i}{\partial x}, \\
\frac{L_{s h}}{N_{c}} \frac{\partial i_{s h}}{\partial t}+\frac{R}{N_{c}} i_{s h} & =v(x)-v_{B},
\end{aligned}
$$

where $v, i$, and $i_{\text {sh }}$ represent the terminal voltage of $I_{D}$, the series current, and the shunt current at $x$, respectively (see appendix for explicit derivations). Using an auxiliary variable $w$, equations (1)-(3) are simplified as follows:

$$
\frac{\partial}{\partial t}\left(\begin{array}{c}
w \\
v
\end{array}\right)=\left(\begin{array}{c}
\frac{1}{L C}\left\{-\left(C R+L \frac{d I_{D}}{d v}\right) w+\frac{1}{N_{c}^{2}} \frac{\partial^{2} v}{\partial x^{2}}-v+v_{B}-R I_{D}\right\} \\
w
\end{array}\right)
$$

The trigger wave $[1,10,11]$ or dissipative solitons $[3,5,12]$ including the pulse under investigation have no velocity flexibility. Then, according to the standard description, the solutions of equation (4) are assumed to be as follows:

$$
\begin{aligned}
& w=W\left(x-c t-x_{1}\right)+W\left(x-c t-x_{2}\right)+b_{w}(x-c t, t), \\
& v=v_{B}+V\left(x-c t-x_{1}\right)+V\left(c x-c t-x_{2}\right)+b_{v}(x-c t, t),
\end{aligned}
$$

where $V$ and $W$ are the voltage and its temporal derivative of the self-sustained pulse, $b_{v, w}$ represents the small overlap function, and $c$ is the pulse velocity. In the following, any function $X(x-$ $\left.c t-x_{i}\right)$ is abbreviated as $X_{i}$ (for $\left.i=1,2\right)$ for brevity.
By substituting equations (5) and (6) into equation (4), the first-order dynamic equations for $b_{w, v}$ are derived as follows:

$$
\frac{\partial b}{\partial t}=M b+g
$$




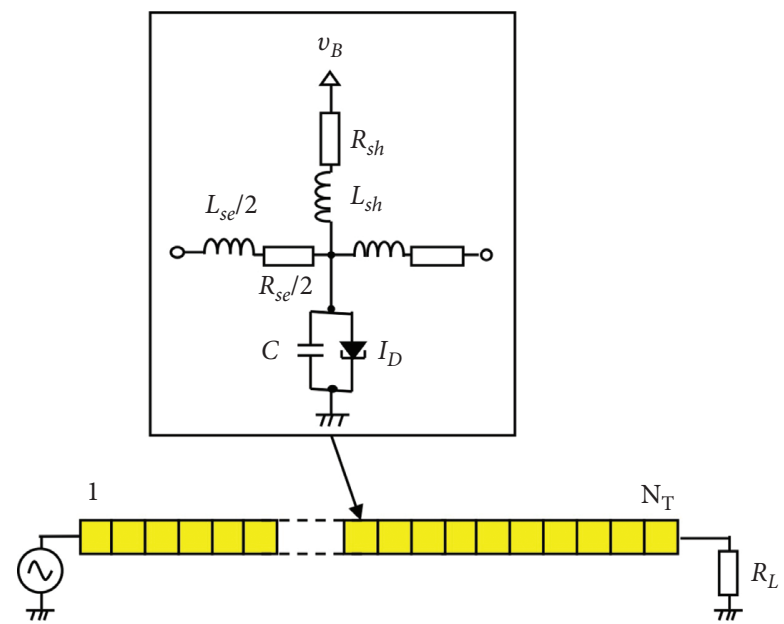

Figure 1: A TD-oscillator lattice under investigation. The input voltage source excites a self-sustained solitary pulse in the TD-oscillator lattice. The resistance $R_{L}$ is a load representing the subsequent circuit.

where $b=\left(b_{w}, b_{v}\right)^{T}$ and the $2 \times 2$ operator $M$ is defined as

$$
M=\left(\begin{array}{c}
\frac{-C R-L G\left(v_{B}+V_{1}\right)+c C L \partial_{x}}{C L} \frac{-1-R G\left(v_{B}+V_{1}\right)-L K\left(v_{B}+V_{1}\right) W_{1}+N_{c}^{-2} \partial_{x}^{2}}{C L} \\
1
\end{array}\right),
$$

where $G=I_{D}^{\prime}$ and $K=G^{\prime}$ ( represents the derivative with respect to the argument). In addition, the inhomogeneous term $g$ is given as follows:

$$
\begin{aligned}
\mathbf{g}= & \left(\begin{array}{c}
g_{1} \\
g_{2}
\end{array}\right) \\
g_{1}= & \frac{-R I_{D}\left(v_{B}+V_{12}\right)-V_{12}-C R W_{12}-L G\left(v_{B}+V_{12}\right) W_{12}+c C L \partial_{x} W_{12}+N_{c}^{-2} \partial_{x}^{2} V_{12},}{C L} \\
& +\dot{x}_{1} \partial_{x} W_{1}+\dot{x}_{2} \partial_{x} W_{2}, \\
g_{2}= & W_{12}+\left(c+\dot{x}_{1}\right) \partial_{x} V_{1}+\left(c+\dot{x}_{2}\right) \partial_{x} V_{2},
\end{aligned}
$$

where $X_{12}$ abbreviates $X_{1}+X_{2}$ for $X=V, W$. The second pulse is assumed to be far apart from the first. Then, the contributions of the second pulse in $M$ can be neglected.
The solvability condition is given by $\langle\Psi, g\rangle=0$ [2], where $\Psi=\left(\psi_{1}, \psi_{2}\right)^{T}$ is the zero eigenvector of the adjoint of M.

$$
M^{\dagger} \Psi=\left(\begin{array}{cc}
\frac{-C R-L G\left(v_{B}+V_{1}\right)-c C L \partial_{x}}{C L} & 1 \\
\frac{-1-R G\left(v_{B}+V_{1}\right)-L K\left(v_{B}+V_{1}\right) W_{1}+N_{c}^{-2} \partial_{x}^{2}}{C L} & -c \partial_{x}
\end{array}\right)\left(\begin{array}{l}
\psi_{1} \\
\psi_{2}
\end{array}\right)=0 .
$$


The resulting equations are as follows:

$$
\begin{aligned}
\psi_{2} & =\left(\frac{R}{L}+\frac{G\left(V_{1}\right)}{C}\right) \psi_{1}+c \partial_{x} \psi_{1}, \\
c C L \partial_{x} \psi_{2} & =\left(-1-R G\left(v_{B}+V\right)_{1}-L K\left(v_{B}+V_{1}\right) W_{1}\right) \psi_{1}+N_{c}^{-2} \partial_{x}^{2} \psi_{1} .
\end{aligned}
$$

First, the solutions $W_{1}$ and $V_{1}$ are numerically obtained by solving equation (4). These solutions are spatially shifted to obtain $W_{2}$ and $V_{2}$. Then, the zero adjoint eigenvector $\Psi^{(1)}=\left(\psi_{1}^{(1)}, \psi_{2}^{(1)}\right)^{T}$ is obtained by solving

$$
\partial_{x}\left(\begin{array}{c}
\phi \\
\psi_{1}^{(1)}
\end{array}\right)=\left(\begin{array}{c}
\frac{c\left(C R+L G\left(v_{B}+V\right)_{1}\right) \phi+\left(1+R G\left(v_{B}+V_{1}\right)\right) \psi_{1}^{(1)}}{N_{c}^{-2}-c^{2} C L} \\
\phi
\end{array}\right)
$$

It is obtained by substituting equation (11) into (12) and introducing the auxiliary function $\phi$.

The solutions $V, W$, and $\Psi^{(1)}$ are used to evaluate the solvability condition $\langle\Psi, g\rangle=0$. The dynamical equations of the pulse positions $x_{1,2}$ are given as follows:

$$
\begin{aligned}
& \gamma_{0} \dot{x}_{1}+\gamma(-l) \dot{x}_{2}+F(l)=0, \\
& \gamma(l) \dot{x}_{1}+\gamma_{0} \dot{x}_{2}+F(-l)=0,
\end{aligned}
$$

where $l \equiv x_{1}-x_{2}$ and

$$
\begin{aligned}
\gamma_{0}= & \int_{-\infty}^{\infty} \mathrm{d} x\left(\psi_{1}(x) \partial_{x} W(x)+\psi_{2}(x) \partial_{x} V(x)\right), \\
\gamma(l)= & \int_{-\infty}^{\infty} \mathrm{d} x\left(\psi_{1}(x+l) \partial_{x} W(x)+\psi_{2}(x+l) \partial_{x} V(x)\right), \\
F(l)= & -\frac{1}{L C} \int_{-\infty}^{\infty} \mathrm{d} x \psi_{1}(x)\left(R \left(I_{D}\left(v_{B}+V(x)+V(x+l)\right),\right.\right. \\
& \left.-I_{D}\left(v_{B}+V(x)\right)-I_{D}\left(v_{B}+V(x+l)\right)\right), \\
& +L\left(G\left(v_{B}+V(x)+V(x+l)\right)-G\left(v_{B}+V(x)\right)\right) W(x), \\
& \left.+L\left(G\left(v_{B}+V(x)+V(x+l)\right)-G\left(v_{B}+V(x+l)\right)\right) W(x+l)\right) .
\end{aligned}
$$
follows:

Finally, equations (16) and (17) are reformulated as

$$
i=-\frac{\left(\gamma_{0}+\gamma(l)\right) F(l)-\left(\gamma_{0}+\gamma(-l)\right) F(-l)}{\gamma_{0}^{2}-\gamma(l) \gamma(-l)} \equiv Z(l) .
$$

$Z(l)$ can be evaluated by setting $N_{c}, L, C$, and $R$ to 30,10 $\mu \mathrm{H}, 500 \mathrm{pF}$, and $5 \Omega$, respectively. Furthermore, the following phenomenological model was employed for $I_{D}$ to simulate the TD's fundamental properties including the presence of the peak and valley voltages and the relatively small negative differential resistance:

$$
\begin{aligned}
I_{D}(V)= & 0 \cdot 005\left(0 \cdot 175 \log \left(\frac{(1+\exp (-0 \cdot 621+54 V))}{(1+\exp (-0 \cdot 621-54 V))}\right)\right. \\
& \left.\times\left(1 \cdot 4+\tan ^{-1}(1 \cdot 85-19 V)\right)+0.0018(\exp (11.6 V)-1)-0 \cdot 187\right),
\end{aligned}
$$

where the numerical values were optimized to simulate the NEC 1S1763 Esaki diode.
In Figure $2 Z(l)$ is plotted for $l \in[-2,2]$. Obviously, $Z(l)$ is antisymmetric with respect to the vertical zero-crossing 


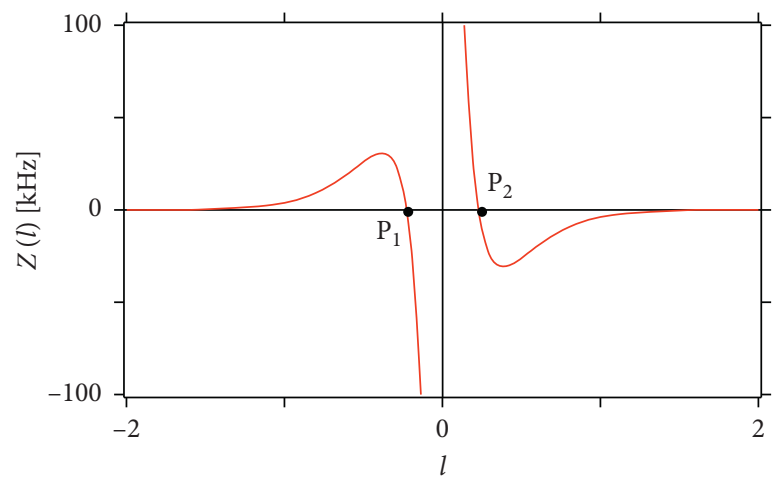

Figure 2: Reduced dynamics of two pulses. Since $i$ is measured on the vertical axis, the pulses asymptotically arrive at either $P_{1}$ or $P_{2}$, regardless of their initial position.

axis, and two stable equilibrium points $P_{1,2}$ exist, corresponding to $l= \pm 0.2275 \equiv l^{*}$. If $l$ is slightly smaller than $P_{2}$, a positive $i$ means that the two pulses interact repulsively, whereas when $l$ becomes larger than $P_{2}$, a consequent attractive interaction diminishes $l$. Therefore, the two successive solitary pulses tend to move in the lattice with a fixed separation $l^{*}$.

To examine this property in a practical discrete lattice, the original lattice equations given in equations (A.1)-(A.3) were solved in the time domain by using the 4 th-order Runge-Kutta method. The results are presented in Figure 3. Equation (22) was used for calculating $I_{D}$. A 200-cell lattice was calculated using $L=4 \mu \mathrm{H}$ and $C=500 \mathrm{pF}$. Two solitary pulses were inserted into the near end of the lattice, and the variation of their temporal separation between peaks was monitored. Initially, $R$ was set to $4 \Omega$. The calculated results are shown in Figures 3(a) and 3(c). The waveform of the self-sustained pulse is shown in Figure 3(a). A long and exponential tail can be observed. Figure 3(c) shows the spatial variation of the pulse separation for nine different values of initial temporal separation, starting from $0.5 \mu \mathrm{s}$ and ending at $1.2 \mu \mathrm{s}$ with increments of $0.1 \mu \mathrm{s}$. For initial separations of less than $1.0 \mu \mathrm{s}$, the spatial separation increases with the moving distance. This is consistent with the reduced model prediction, which states that any two pulses with small separation are subject to repulsive interaction. On the other hand, Figures 3(b) and 3(d) correspond to the case of $R$ being zero. The temporal waveform in Figure 3(b) exhibits an oscillatory tail, and correspondingly, several different converging separations exist, as shown in Figure 3(d). An effort was made to characterize this loss-free lattice using the reduced model. However, equation (15) fails to converge to the required solution.

Note that no plots are shown for initial separations of less than $0.5 \mu \mathrm{s}$ in either case. When the spacing becomes too small, the subsequent pulse is affected by the voltage drop caused by the tail of the leading pulse and decays and disappears gradually.

\section{Experimental Results}

The nontrivial motion of two solitary pulses was examined by fabricating a test lattice on breadboards. The $L$ and $C$ values were set to $5.0 \mu \mathrm{H}$ and $2.0 \mathrm{nF}$, respectively. No resistors were used for $R$. Instead, the parasitic $L$ resistance values were used. An NEC 1S1763 Esaki diode was used to implement the TDs. The total number of connected cells $N_{L}$ was 50. A Keysight 1134B active probe was used to detect the time domain voltage variations in all cells of the lattice using an Agilent DS90254A oscilloscope.

The measured variation of the pulse separation is shown in Figure 4. The voltages were measured at nine different points (from the 1 st cell to the 46 th cell with increments of 5 cells) for an initial temporal separation between pulses varying from 0.0 to $6.0 \mu \mathrm{s}$ with increments of $0.055 \mu \mathrm{s}$. The dependence of temporal waveforms based on the initially assigned temporal delays was measured in the first and 26th cells. The results are plotted in Figures 4(a) and 4(b), respectively. At the point of incidence, the leading pulse occupies a common temporal position, whereas the subsequent pulse delays linearly from the bottom (no separation) to the top (6.0- $\mu$ s separation). In contrast, in the 26th cell, the second pulse disappears for an initial delay of less than $0.5 \mu$ s. The pulse delay exhibits only a small variation for an initial delay greater than $2.2 \mu \mathrm{s}$. For other initial delay values, the second pulse survives, and its delay tends to converge to a common value (Figure 4(c)). Both the deceleration of the second pulse and the acceleration of the leading pulse contribute to a saturating delay. This observation indicates that mutual interaction is established between the pulses.

The pulse dynamics are shown in Figure 5. This figure illustrates the variation of the pulse-to-peak separation, where the initial pulse separation ranges in $0.71-1.67 \mu \mathrm{s}$ with increments of $0.055 \mu \mathrm{s}$. Beyond the 31 st cell, the pulses with initial separation of less than $1.2 \mu \mathrm{s}$ exhibit a similar separation trend. However, the separation convergence value is not kept fixed. Instead, it keeps increasing with the distance traveled. Moreover, beyond the 46th cell, the second pulse gradually decays and finally disappears. This insufficiency may be due to fluctuation in device parameter values and breadboard's bandwidth. A complete validation requires a thorough optimization in the selected parameter values and platform.

To simulate the measured results several Runge-Kutta calculations were carried out. The device parameter values were fluctuated with a $10 \%$ standard deviation. The average values of $L, C$, and $R$ were set to $5 \mu \mathrm{H}, 2 \mathrm{nF}$, and $1 \Omega$, 


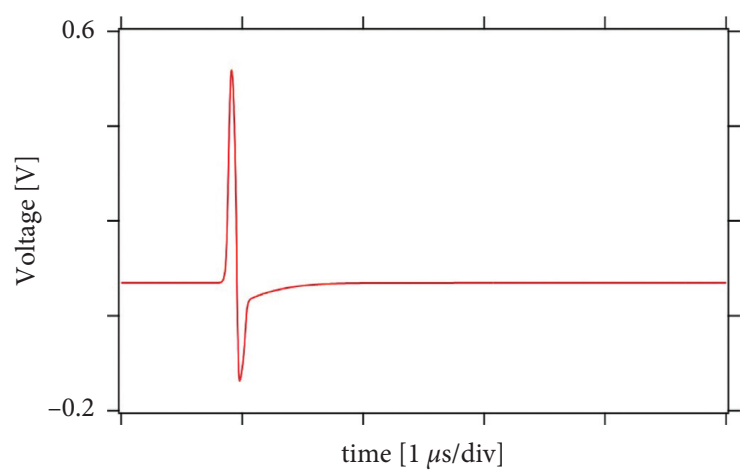

(a)

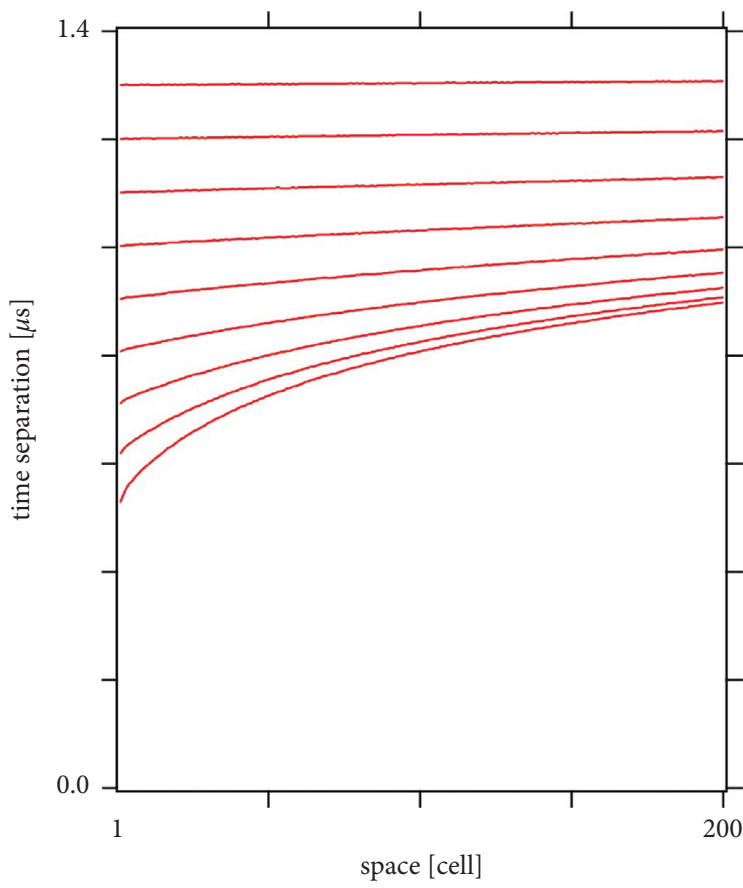

(c)

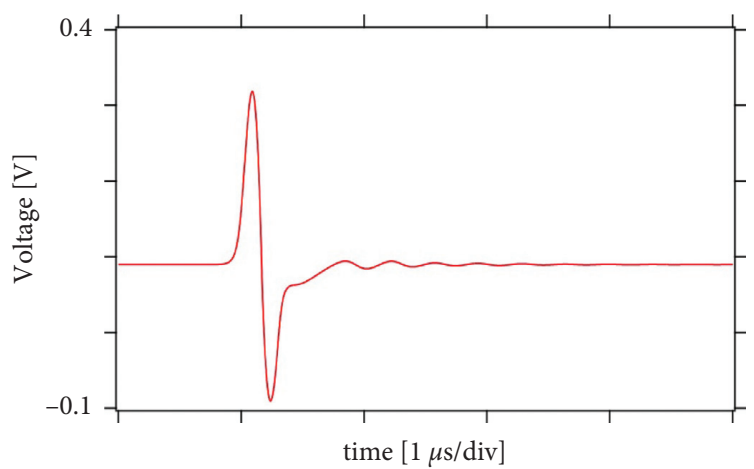

(b)

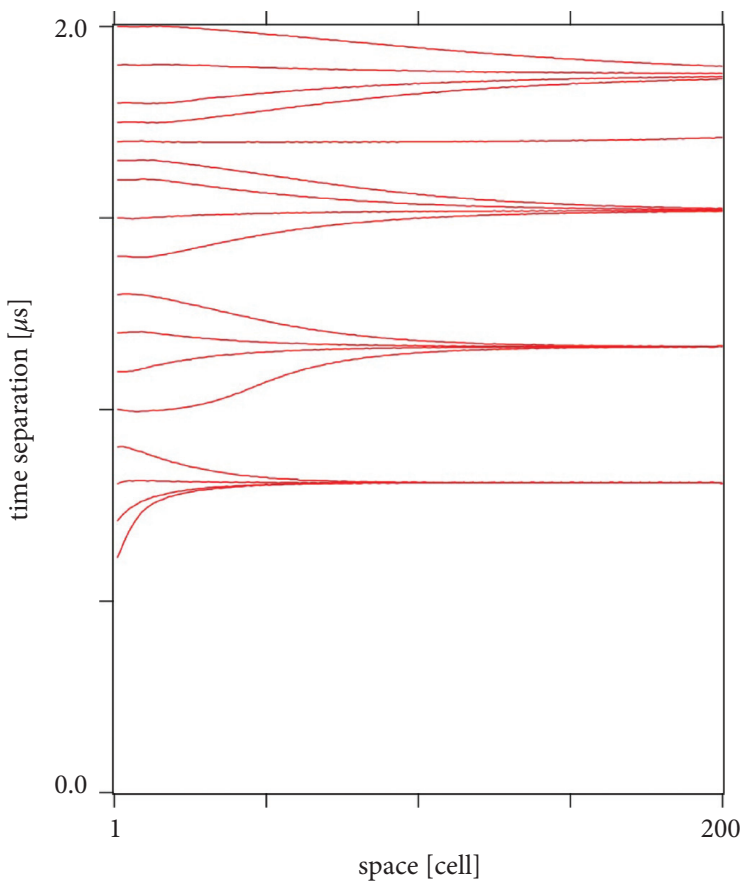

(d)

FIGURE 3: Interpulse separation predicted by Runge-Kutta analyses. The unit cell contains (a) 4- $\Omega$ resistors and (b) no resistors.

respectively. Triangular pulses were applied to the near end with a $50 \Omega$ input resistor, and the far end was terminated with a $50 \Omega$ load resistor. The incident pulse had $100 \mathrm{~ns}$ fall and rise times and $0.5 \mathrm{~V}$ amplitude. Figure 6(a) compares the measured and calculated waveforms. The waveforms commonly have the short positive main pulse with a negative long tail. However, the measured pulse does not return to the zero level unlike the calculated one as indicated by arrows. This negative offset of the measured pulse may be caused by some technical problem. For example, the main pulse was steep sufficiently to exceed the breadboard's bandwidth for reliable operation. Thus, we consider the numerical model under investigation as being suitable to simulate a well-composed TD-oscillator lattice. Figure 6(b) shows the calculated spatial waveform of the self-sustained pulse moving to the right. The pulse's spatial width is estimated to be 17 cells with the 5-cell main pulse and 12cell tail.
To examine the double-pulse response two triangular pulses are input to the near end of a 500-cell lattice. The initial pulse separation $t_{s}$ was set to $0.63 \mu \mathrm{s}$ (the second pulse disappeared for $\left.t_{s} \leq 0.62 \mu \mathrm{s}\right)$. The spatiotemporal behavior of two pulses is shown in Figure 7(a). The separation between the first and second pulses increases monotonically with decreasing growth rate (its spatial variation is shown in Figure 7(b)). The presence of the first pulse tail decelerates the second pulse. The almost asymptotic spatial waveform is shown in Figure 7(c), which was detected at the time indicated by the dashed line in Figure 7(a). The spatial width is estimated to be 34 cells, which is just twice as wide as that of the single pulse. Thus, the separation tends to increase until the pulses' overlap is completely eliminated. The lattice size must be set more than 34 cells for the coexistence of two independent pulses.

Figure 8 shows the calculated results when three evenly separated pulses are input to the 500 -cell lattice $\left(t_{s}=0.63 \mu \mathrm{s}\right)$. 


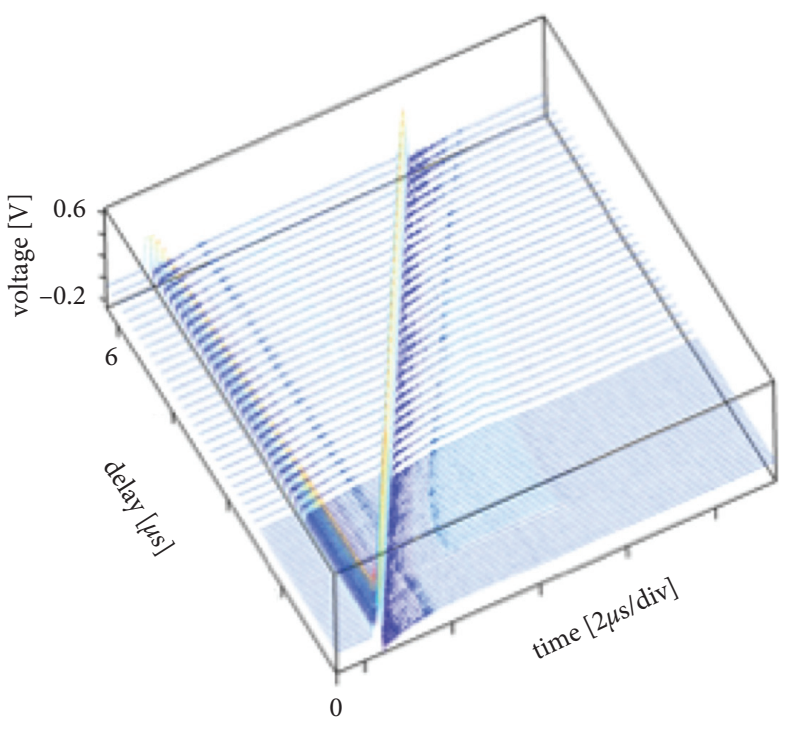

(a)

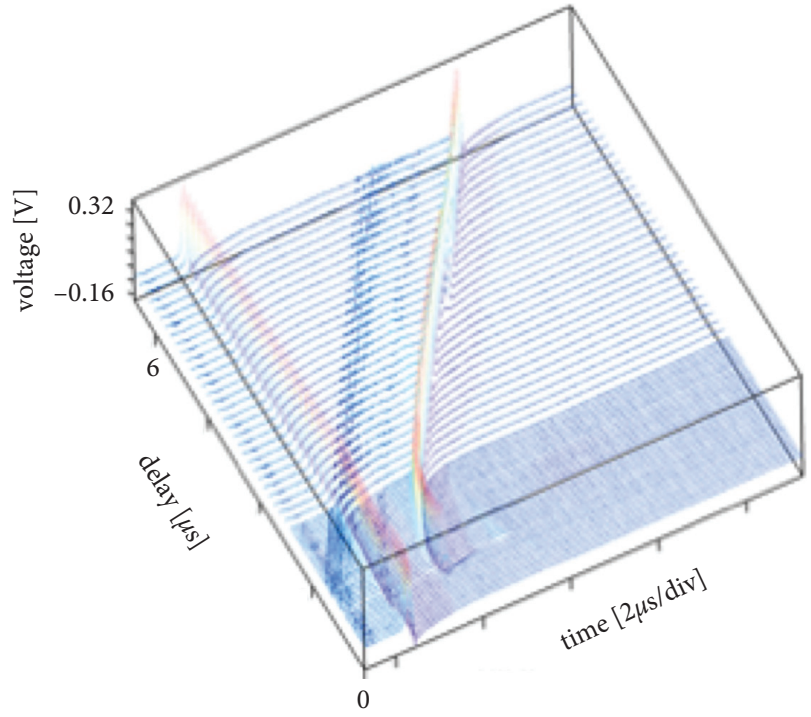

(b)

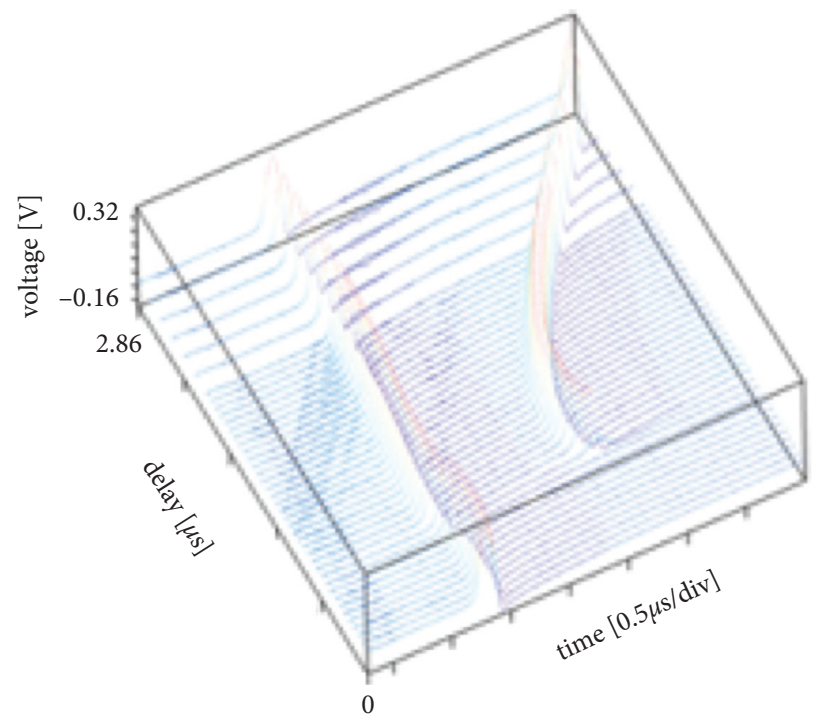

(c)

FIgURE 4: The measured temporal evolution of interpulse temporal separation. The temporal waveforms for several different initial delays detected in (a) the first cell and (b) the 26th cell. (c) An enlarged view of the waveforms detected in the 26th cell for small interpulse delays.

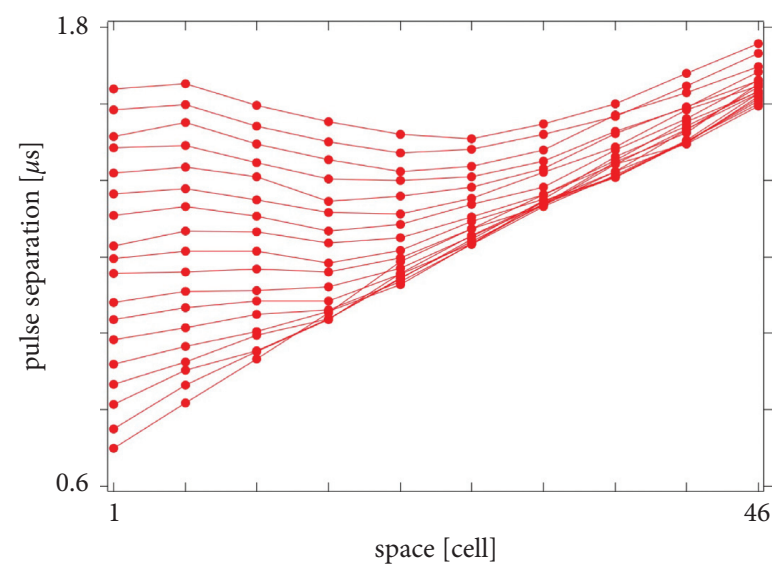

FIgURE 5: Spatiotemporal variation of pulse separation. 


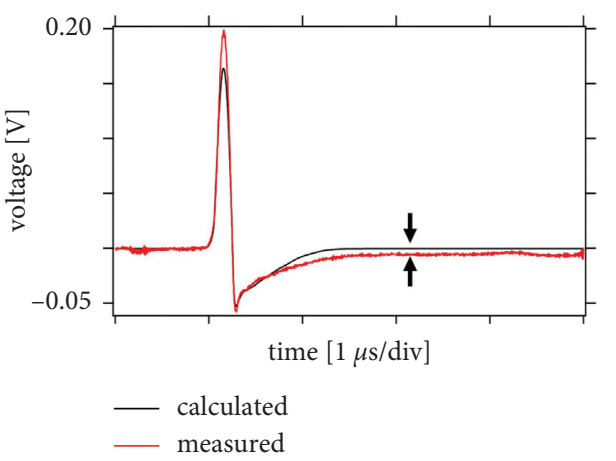

(a)

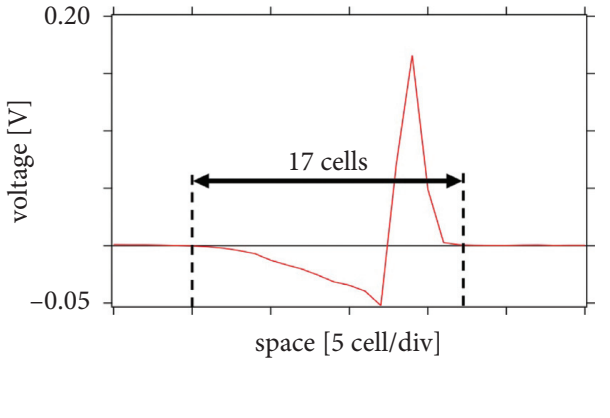

(b)

Figure 6: Properties of calculated pulse. (a) The comparison between the measured and calculated waveforms and (b) the spatial waveform of the calculated pulse.

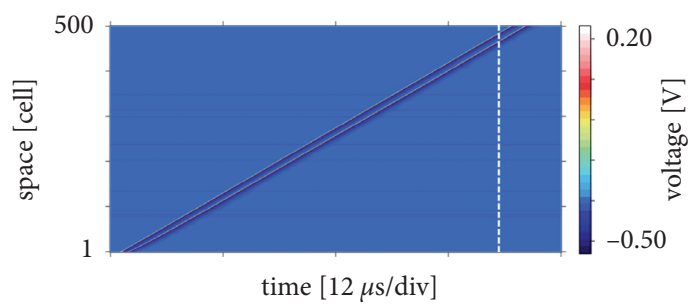

(a)

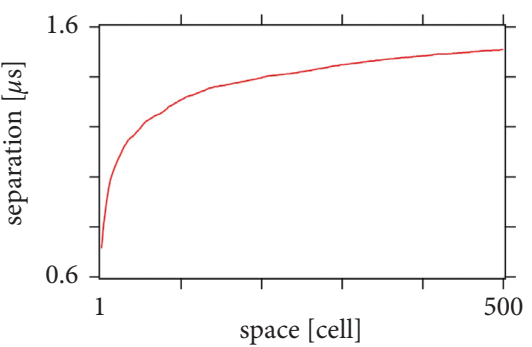

(b)

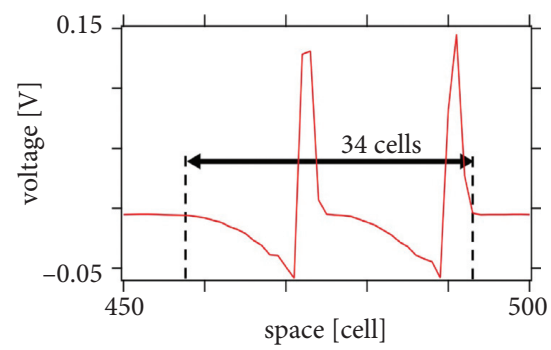

(c)

FIGURE 7: Calculated lattice's response to the double-pulse incidence. (a) The spatiotemporal voltage map, (b) the spatial variation of the pulse separation, and (c) typical spatial waveform.

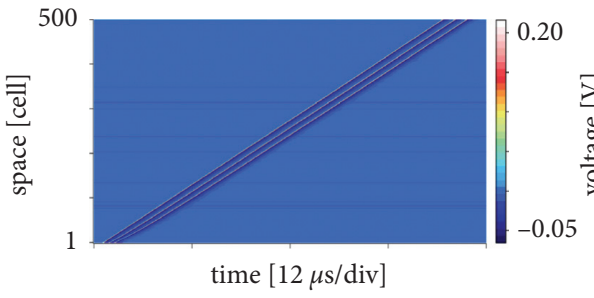

time $[12 \mu \mathrm{s} / \mathrm{div}]$

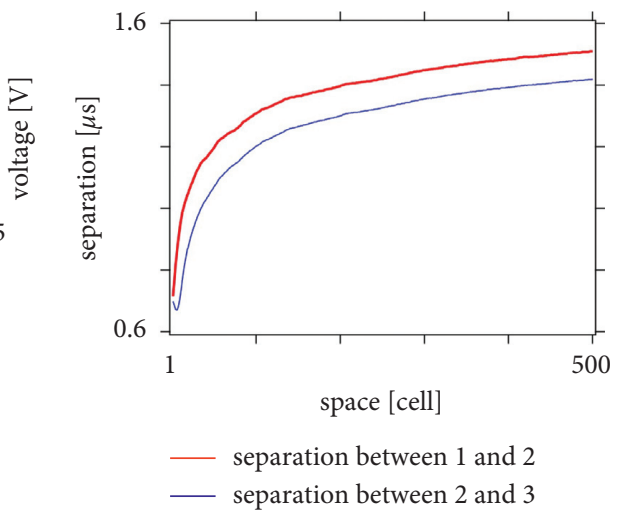

(b)

FIGURE 8: Calculated lattice's response to the triple-pulse incidence. (a) The spatiotemporal voltage map and (b) the spatial variation of the pulse separation (red: the separation between pulses 1 and 2, blue: the separation between pulses 2 and 3 ). 
The spatiotemporal voltage map shown in Figure 8(a) shows that three pulses move in the lattice and reach the far end without being eliminated on the way. Furthermore, the pulses 1 and 2 become more distant and the separation growth rate is independent of the presence of pulse 3 . This is clearly observed in Figure 8(b), where the spatial variation of the interpulse separations is plotted. The red curves in Figures 7(b) and 8(b) are almost coincident. The separation between pulses 2 and 3 (blue curve) also has the increasing tendency. However, it initially decreases slightly and is always smaller than its counterpart up to the far end. The discrepancy in separations may start to decrease only after the overlap between pulses 1 and 2 becomes negligibly small.

To clarify how the number of advancing pulses affects the evolution of the pulse separation we examined the lattice response to a pulse train. The lattice size was reduced to 400 for convenience. As a result, the device fluctuation differs from that used in the 500-cell lattice. A train of evenly separated triangular pulses $\left(t_{s}=1.0 \mu \mathrm{s}\right)$ was input to the near end. Every pulse reaches the far end as shown in Figure 9(a). For brevity, the temporal separation between the $m$ th and $(m+1)$ th pulses is simply denoted as the $m$ th separation. Figure 9(b) shows the spatial dependence of the $m$ th separation $(1 \leq m \leq 31)$. At any position in the lattice the $(m+1)$ th separation becomes smaller than the $m$ th one for $m<20$. Furthermore, the separation preserves its initial value even at the far end for $m \geq 20$. As a whole, the increasing separation is caused by the presence of the 1st pulse, i.e., the temporal edge of the pulse train. This edge effect gradually percolates through to the interior pulses at the rate of 20 cells/pulse (twenty pulses from the edge is subject to the edge effect by 400-cell propagation in the lattice). Without such an edge an input pulse train preserves its interpulse separation even in the lattice. This may be realized through the balance in multiple-pulse interactions. Figure 9(c) shows the spatial waveform of the pulse train preserving initial separation. The spatial period is estimated to be 11 cells, being much smaller than the width of the single pulse. It may be reduced to be in the neighborhood of the minimum value below which the following pulse disappears. It may also be expected that the pulse interaction equalizes the separation of each neighboring pulse pair. In the following, we consider two pulses in a lattice loop, which simulates the steadily moving pulse train in a straight lattice, to examine whether the equalization of pulse separation is feasible or not.

\section{Application}

An application utilizing pulse interaction is the phase-noise reduction. The case where two or more solitary pulses rotate in a lattice loop is considered. By implementing the multiple output ports, the device can operate as a multiphase oscillator [13-17], which contributes to the relaxation of the base frequency to drive the ultrafast systems operating in submillimeter wave or terahertz frequencies using timeinterleaving [18] or output combining [19].

The oscillation frequency increases with the number of rotary pulses. However, the fluctuation in the position of each pulse may result in degradation in the oscillator's phase-noise performance. The repulsive interaction observed can force rotary pulses to be equally spaced, contributing to phase-noise reduction.

In a loop with size $L_{0}$, the same equation for $l_{2}=L_{0}-l$ holds as $l$ in equation (19). Thus the following equation is obtained:

$$
-i=-\frac{\left(\gamma_{0}+\gamma\left(L_{0}-l\right)\right) F\left(L_{0}-l\right)-\left(\gamma_{0}+\gamma\left(l-L_{0}\right)\right) F\left(l-L_{0}\right)}{\gamma_{0}^{2}-\gamma\left(L_{0}-l\right) \gamma\left(l-L_{0}\right)} .
$$

By subtracting equation (21) from equation (19), $l$ satisfies the following equation:

$$
\begin{aligned}
i= & \frac{1}{2}\left(-\frac{\left(\gamma_{0}+\gamma(l)\right) F(l)-\left(\gamma_{0}+\gamma(-l)\right) F(-l)}{\gamma_{0}^{2}-\gamma(l) \gamma(-l)}\right. \\
& \left.+\frac{\left(\gamma_{0}+\gamma\left(L_{0}-l\right)\right) F\left(L_{0}-l\right)-\left(\gamma_{0}+\gamma\left(l-L_{0}\right)\right) F\left(l-L_{0}\right)}{\gamma_{0}^{2}-\gamma\left(L_{0}-l\right) \gamma\left(l-L_{0}\right)}\right) \equiv Z_{L_{0}}(l) .
\end{aligned}
$$

Notice that the equilibrium point of equation (22) occurs for $l= \pm L_{0} / 2$. To quantify the pulse dynamics, the same lattice parameter values and $I_{D}$ model presented in Figure 2 were employed. The results shown in Figure 10(a) correspond to the case of $L_{0}$ being set to 1.0. Two stable equilibrium points $P_{1,2}$ and an unstable one $Q$ corresponding to the half-loop are observed. Since the half-loop size exceeds $l^{*}$, two pulses tend to move in the loop with an $l^{*}$ separation as that in the straight lattice. On the other hand, the case of $L_{0}$ being set to 0.5 is shown in Figure 10(b), where $Q$ becomes stable. If the loop size is designed to be smaller than $l^{*}$ a novel strategy for reducing phase noise in coupled oscillator systems may emerge.
The equalization of pulse separation in a TD lattice loop was demonstrated by carrying out a Runge-Kutta calculation for the structure shown in Figure 11(a). The $L, R$, and $C$ values were set to $10.0 \mu \mathrm{H}, 5 \Omega$, and $2.0 \mathrm{nF}$, respectively. Two 10 -cell straight lattices were prepared for the signal input (orange) and output (green) signals. In addition, two coupled TD lattices (yellow) were implemented to introduce and extract self-sustained pulses in the loop. One of the coupled lattices was connected to the input/output lattices, and the other partially formed the lattice loop with the supplementary 26-cell lattice (blue). The coupling capacitance $C_{c p l}$ was set to $470 \mathrm{pF}$. When two pulses were inserted into the input lattice, they started to rotate in the loop in an 

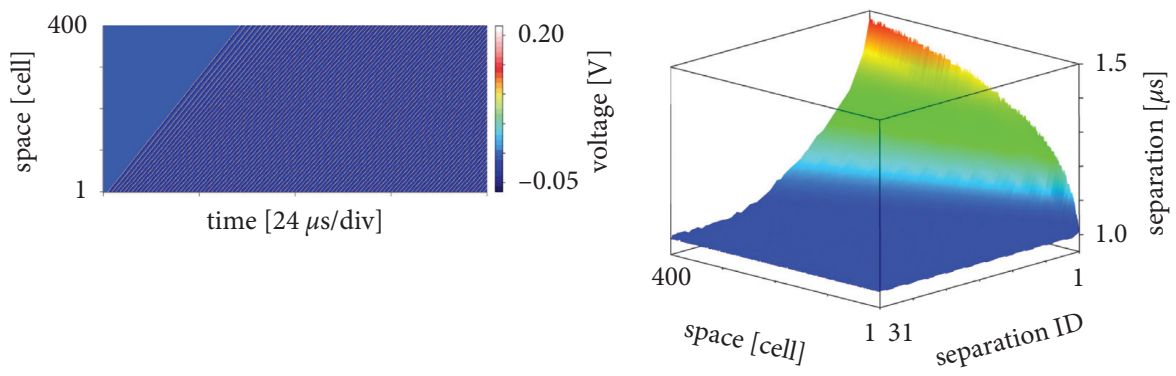

(a)

(b)

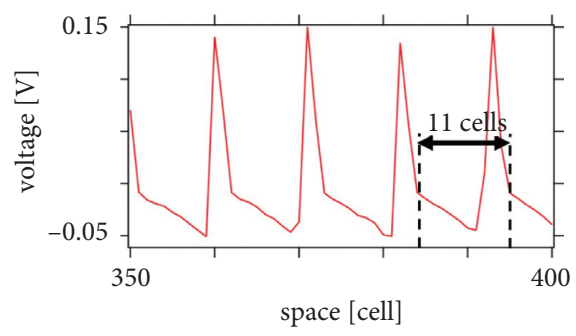

(c)

Figure 9: Calculated lattice's response to the multiple-pulse incidence. (a) The spatiotemporal voltage map, (b) the variation of the pulse separation with respect to the space and separation ID, and (c) the spatial waveform of the steadily moving periodic pulse train. The separation between the $i$ th and $(i+1)$ th pulses has the separation ID of $i$ in Figure 9(b).

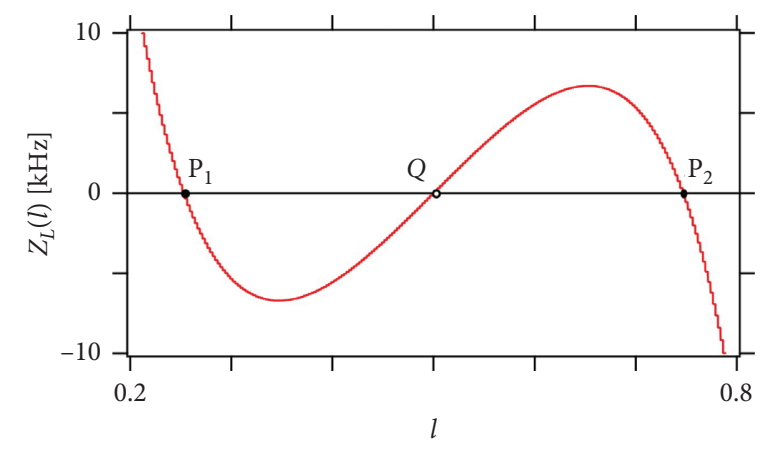

(a)

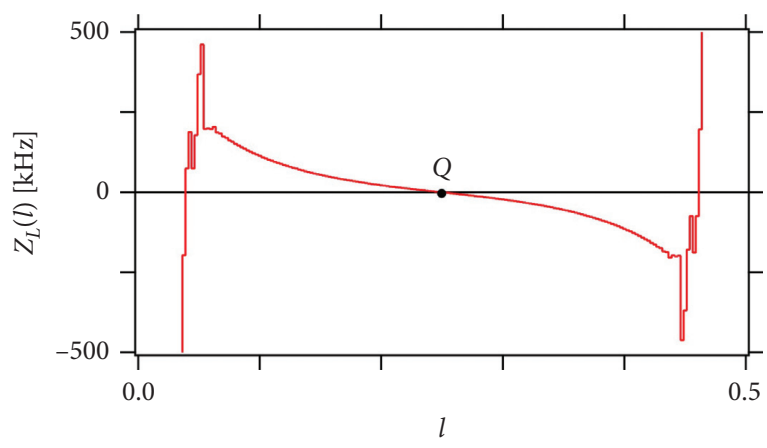

(b)

Figure 10: Predictions of pulse reduction in a loop. The loop size is set to (a) 1.0 and (b) 0.5 .

anticlockwise fashion. The repeated rotation of these two pulses in the loop results in the pulse train at the OUT port. The separation variation of the two pulses with the rotation was observed. Figure 11(b) shows the trajectories of the two rotary pulses in the lattice loop. Time progresses horizontally, and the spatial position is measured on the vertical axis. The vertical axis bottom and top correspond to the first and 36th cells, respectively. Due to the closed structure, the pulse reaches the first cell after passing the 36th cell, so that the tilted stripes in Figure 11(b) represent one or the other rotary pulses, alternatively. Initially, the pulse separation set was rather unbalanced. At the beginning, the first pulse leads the second one, as shown by $P_{1}$, which is relatively larger than $P_{2}=36-P_{1}$. After a six-time rotation, the separation becomes $Q_{1}$, which is equal to $Q_{2}=36-Q_{1}$. Thus, the pulse separation is successfully and promptly equalized.
To quantify the phase-noise reduction achieved using repulsive interactions, we obtained the perturbation projection vector (PPV) [20, 21] or phase sensitivity for the oscillation on the TD lattice loop. The lattice equations shown in eqs. (A.1)-(A.3) were solved for the TD-oscillator lattice loop with $N_{c}=30$. The $L, C$, and $R$ values were set to $10 \mu \mathrm{H}, 2 \mathrm{nF}$, and $5 \Omega$, respectively. No parameter fluctuations were assumed. Equations (A.1)-(A.3) are conveniently written as follows:

$$
\frac{\mathrm{d} X}{\mathrm{~d} t}=F(X)
$$

where the first, second, and last $N_{c}$ components of the $3 N_{c}$ vector $X$ correspond to $\left\{i_{n}\right\},\left\{v_{n}\right\}$, and $\left\{j_{s h, n}\right\}$, respectively. The components of $F$ are given by the right-hand side of equations (A.1)-(A.3) with proper variable replacement. The 


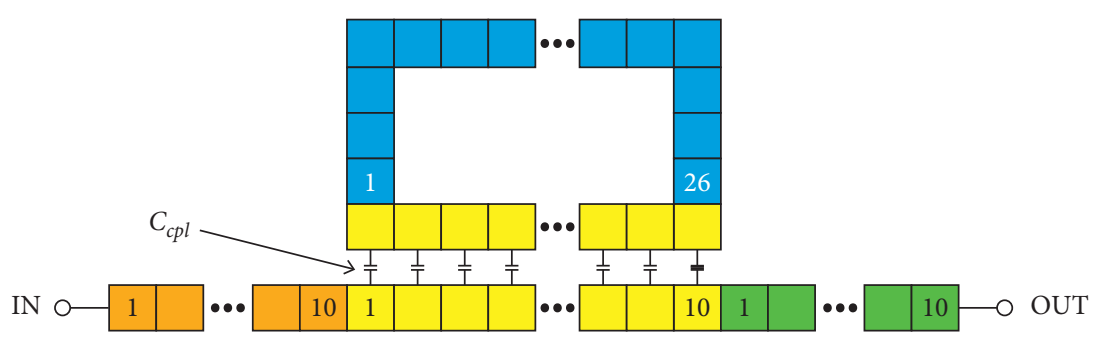

(a)

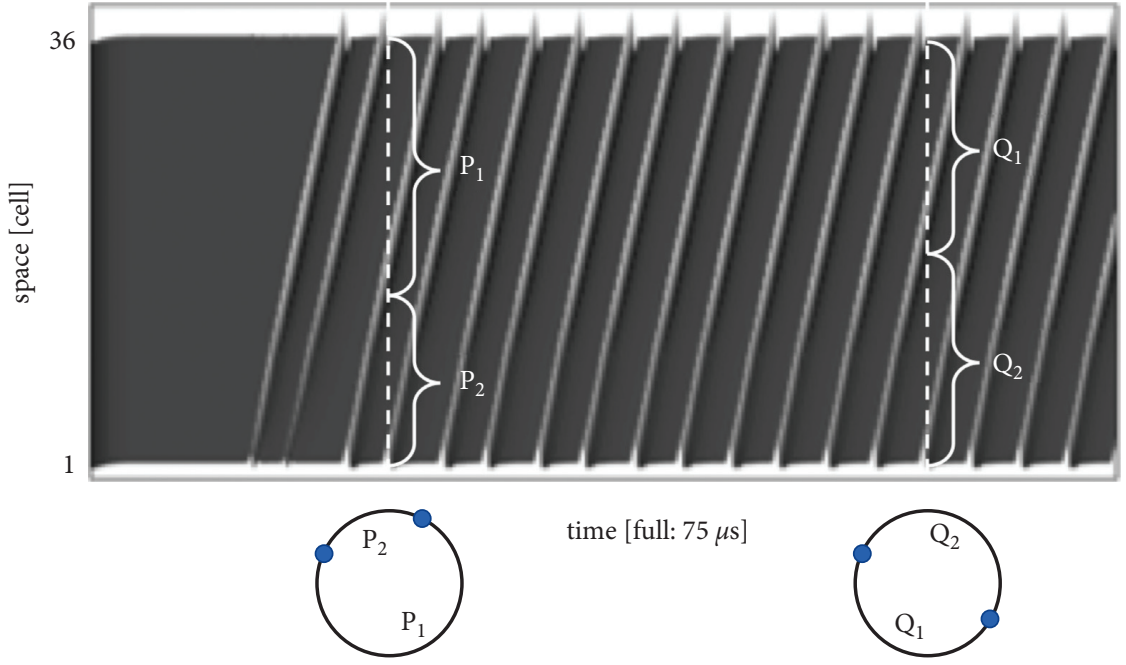

(b)

Figure 11: Numerically obtained pulse dynamics in a tunnel-diode (TD) lattice loop. (a) Structure of the TD lattice loop and interface lattices and (b) calculated spatiotemporal voltage map.

phase-noise performance is significantly dependent on which noise source mainly influences it. In this study, the white noise voltage source was connected in series with $R_{s e, s h}$ in each cell; thus, the number of sources becomes $2 N_{c}$. As a result, the governing equation is given as follows:

$$
\frac{\mathrm{d} X}{\mathrm{~d} t}=F(X)+B \xi
$$

where the $2 N_{c}$ vector $\xi$ enumerates the white noise voltage sources and $B$. represents the $3 N_{c} \times 2 N_{c}$ transfer matrix between $\xi$ and $X$. The deterministic part of equation (24) has a limit cycle solution denoted by $X_{s}$. We then linearized $F$ for $X=X_{s}$ and solved the resulting linear equation to obtain the $3 N_{c} \times 3 N_{c}$ monodromy matrix $\Phi$. The PPV, denoted by $Z$, can be defined as the eigenvector of the adjoint of $\Phi$ corresponding to a zero eigenvalue with an appropriate normalization $[20,21]$. The phase noise is characterized by a unique parameter $c$, defined as follows:

$$
c=\frac{1}{T_{0}} \int_{0}^{T_{0}} \mathrm{~d} t Z^{\dagger}(t) B\left(X_{s}(t)\right) B^{\dagger}\left(X_{s}(t)\right) Z(t),
$$

where $T_{0}$ represents the period of limit cycle oscillation. For sufficiently small $c$, the phase noise of the oscillator can then be calculated using the following equation:

$$
\mathscr{L}\left(f_{m}\right)=10 \log _{10} \frac{f_{0}^{2} c}{f_{m}^{2}}
$$

where $f_{0}$ and $f_{m}$ are the oscillation and offset frequencies, respectively [21].

First, the single, double, and triple rotary pulse solutions were obtained by solving equation (23) in the time domain by using the Runge-Kutta method. Figure 12(a) shows the properties of the single pulse rotation. The upper panel shows the spatiotemporal voltage map. A single pulse is shown to rotate in the loop to the direction the cell address increases. The rotation frequency was $241.6 \mathrm{kHz}$. The lower panel shows the locations of the eigenvalues of $\Phi$. Because the rotary pulse is stable, all eigenvalues are included in the unit circle except for $\lambda=1$ (indicated by black arrow). Figures 12(b) and 12(c) show the properties of the double and triple rotary pulse solutions, respectively. The mismatch in rotation direction does not matter because the lattice was homogeneous. The rotation frequency decreases as the pulse number increases: 235.1 and $215.1 \mathrm{kHz}$ for the double and triple cases, respectively (for comparison, two- and threepulse durations were employed as the oscillation period for the double and triple cases, respectively). The second largest eigenvalue is indicated by green arrows in each lower panel. As suggested in [22], when this eigenvalue approaches the unit circle more, the oscillator's original $Q$ factor increases. 

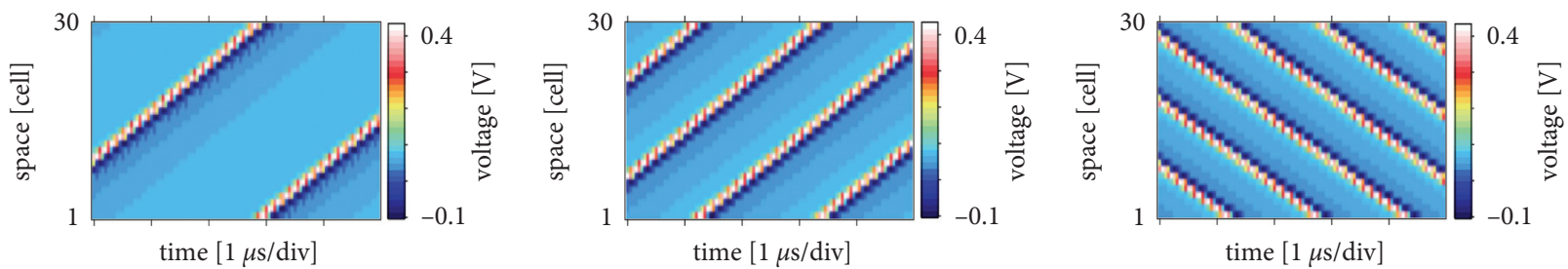

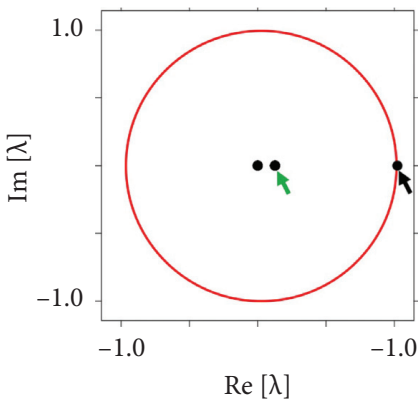

(a)

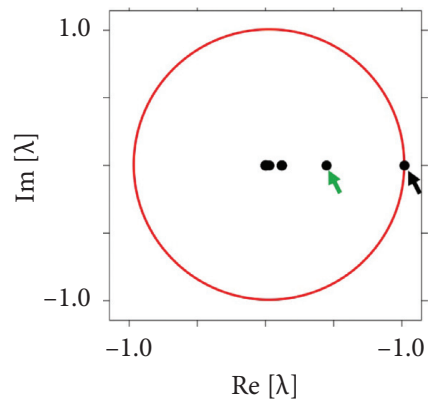

(b)

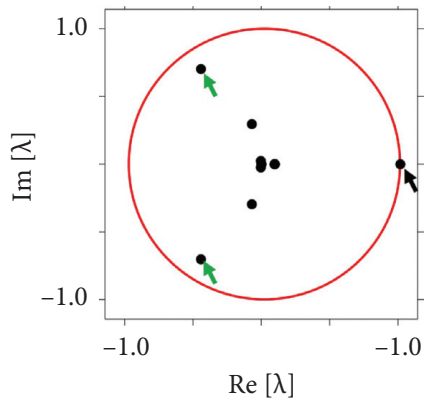

(c)

Figure 12: Eigenvalues of monodromy matrix. (a) Single, (b) double, and (c) triple rotary pulse(s) in the loop. In each figure, the spatiotemporal voltage map is shown at the top and the eigenvalues of the corresponding monodromy matrix are shown at the bottom. For clarity, the vertical and horizontal scales are shown only for the left voltage map, although the other two are shown in the common scale. Red curve shows the unit circle.

The presence of two or three pulses in the loop contributes to the enhanced $Q$ factor.

To evaluate PPV the white noise source intensity for resistance $R$ was assumed to be $\sqrt{4 k_{B} T R \Delta f}$, where the temperature $T$ and the noise frequency bandwidth $\Delta f$ were set to $300 \mathrm{~K}$ and $100 \mathrm{kHz}$, respectively $\left(k_{B}\right.$ : Boltzmann constant). The $c$ value was calculated to be $3.53 \times 10^{-13}, 1.46$ $\times 10^{-13}$, and $1.58 \times 10^{-13} \mathrm{~s}$ for the single, double, and triple cases, respectively. By using equation (26), the phase noise is calculated to be $-76.86,-80.92$, and $-81.37 \mathrm{dBc} / \mathrm{Hz}$ at $1 \mathrm{kHz}$ offset for the single, double, and triple cases, respectively. By the presence of the second pulse, the phase noise was reduced by $4 \mathrm{dBc} / \mathrm{Hz}$. Further investigations are required to optimize the impact of the interpulse repulsion on phasenoise reduction, including the loop size, device parameters, and bias voltage.

As mentioned above the velocity of the self-sustained pulse significantly depends on $v_{B}[1]$; therefore, the rotation frequency also exhibits significant dependence on $v_{B}$. This means that the oscillator under investigation operates as a voltage-controlled oscillator, which can be efficiently implemented in a phase-locked loop (PLL), where the low-frequency phase noise inside the loopback bandwidth can be reduced to the same level as the reference oscillator. The interpulse repulsion promptly equalizes separation; therefore, it contributes to the reduction of the high-frequency phase noise. Furthermore, the present scheme does not disturb the PLL operation and has the synergetic potential to achieve broadband phase-noise reduction.

\section{Conclusions}

The interactions between two successive self-sustained pulses were characterized in a TD-oscillator lattice. The reduction theory, which converts the original circuit equations into the dynamic equations with respect to the pulse positions, predicts that the lossy TD-oscillator lattice has a unique stable point in the pulse separation, if half of the loop size becomes less than the asymptotically converging stable spacing. Furthermore, the antipodal points exhibit unique stable positions for two pulses rotating in the lattice loop. The results obtained from several measurements conducted using a test breadboarded lattice clearly demonstrated that the delay between the two comoving pulses tends to converge to a fixed value. In addition, the interpulse delay equalization was successfully calculated in the time domain. We believe that the lattice loop investigated provides an efficient platform for generating low-phase-noise multiphase oscillatory signals.

\section{Appendix}

Derivation of equations (1)-(3) is as follows.

According to Kirchhoff's law, the following lattice equation is obtained:

$$
\begin{gathered}
L_{s e} \frac{\mathrm{d} i_{n}}{\mathrm{~d} t}+R_{s e} i_{n}=v_{n-1}-v_{n}, \\
C \frac{\mathrm{d} v_{n}}{\mathrm{~d} t}+I_{D}\left(v_{n}\right)+j_{s h, n}=i_{n}-i_{n+1},
\end{gathered}
$$




$$
L_{s h} \frac{\mathrm{d} j_{s h, n}}{\mathrm{~d} t}+R_{s h} j_{s h, n}=v_{n}-v_{B}
$$

where $v_{n}, i_{n}$, and $j_{s h, n}$ represent the voltage at the $n$th cell, the current flowing through $L_{s e}$ from the $n-1$ to the $n$th cells, and the current flowing through $L_{s h}$ from the $n$th cell to $v_{B}$, respectively. Let $N_{c}$ be the cell size corresponding to the unit length. Then, the per-unit-length quantities (represented by small letters) are defined as follows:

$$
\begin{gathered}
c=N_{c} C, \\
l_{s e}=N_{c} L_{s e}, \\
r_{s e}=N_{c} R_{s e}, \\
l_{s h}=\frac{L_{s h}}{N_{c}}, \\
r_{s h}=\frac{R_{s h}}{N_{c}} .
\end{gathered}
$$

By introducing the continuous variable $x$ in place of $n$ and defining the unit-cell length as $\Delta x$, equations (A.1)-(A.3) are approximated as follows:

$$
\begin{aligned}
l_{s e} \Delta x \frac{\mathrm{d} i}{\mathrm{~d} t}+r_{s e} \Delta x i & =v(x-\Delta x)-v(x), \\
c \Delta x \frac{\mathrm{d} v}{\mathrm{~d} t}+I_{D}(v) \Delta x+j_{s h} & =i(x)-i(x+\Delta x), \\
\frac{l_{s h}}{\Delta x} \frac{\mathrm{d} j_{s h}}{\mathrm{~d} t}+\frac{r_{s h}}{\Delta x} j_{s h} & =v-v_{B} .
\end{aligned}
$$

Let $\Delta x$ be negligibly small. Then, equations (A.9)-(A.11) become as follows:

$$
\begin{aligned}
l_{s e} \frac{\partial i}{\partial t}+r_{s e} i & =-\frac{\partial v}{\partial x} \\
c \frac{\partial v}{\partial t}+I_{D}(v)+i_{s h} & =-\frac{\partial i}{\partial x}, \\
l_{s h} \frac{\partial i_{s h}}{\partial t}+r_{s h} i_{s h} & =v-v_{B},
\end{aligned}
$$

where $i_{s h}=j_{s h} / \Delta x$. Equations (1)-(3) are obtained by using per-unit-cell quantities in place of the per-unit-length ones and setting $L_{s e}=L_{s h}=L$ and $R_{s e}=R_{s h}=R$.

\section{Data Availability}

The data that support the findings of this study are available on request from the corresponding author. The data are not publicly available due to privacy or ethical restrictions.

\section{Conflicts of Interest}

The author declares that there are no conflicts of interest.

\section{Acknowledgments}

The author gratefully acknowledges the financial support by JSPS KAKENHI Grant nos. 20K04606 and 21H04546.

\section{References}

[1] K. Narahara, "Self-sustained solitary waves in a tunnel diode oscillator lattice and their applications in frequency division," International Journal of Circuit Theory and Applications, vol. 49, pp. 505-512, 2021.

[2] S.-I. Ei and T. Ohta, "Equation of motion for interacting pulses," Physical Review E-Statistical Physics, Plasmas, Fluids, and Related Interdisciplinary Topics, vol. 50, pp. 4672-4678, 1994.

[3] C. Duprat, D. Tseluiko, S. Saprykin, S. Kalliadasis, and F. Giorgiutti-Dauphiné, "Wave interactions on a viscous film coating a vertical fibre: formation of bound states," Chemical Engineering and Processing, vol. 50, pp. 519-524, 2011.

[4] M. Pradas, D. Tseluiko, and S. Kalliadasis, "Rigorous coherent-structure theory for falling liquid films: viscous dispersion effects on bound-state formation and selforganization," Physics of Fluids, vol. 23, 2012.

[5] D. Tseluiko, "Weak interaction of solitary pulses in active dispersive-dissipative nonlinear media," IMA Journal of Applied Mathematics, vol. 79, pp. 274-299, 2014.

[6] Y. Y. Ruai, Y. Konishi, S. T. Allen, M. Reddy, and M. H. W. Rodwell, "A traveling-wave resonant tunnel diode pulse generator," IEEE Microwave and Guided Wave Letters, vol. 4, pp. 220-222, 1994.

[7] S. G. Muttlak, O. S. Abdulwahid, J. Sexton, M. J. Kelly, and M. Missous, "InGaAs/AlAs resonant tunneling diodes for $\mathrm{THz}$ applications: an experimental investigation," IEEE Journal of the Electron Devices Society, vol. 6, pp. 254-262, 2018.

[8] J. Encomendero, R. Yan, A. Verma et al., "Room temperature microwave oscillations in GaN/AlN resonant tunneling diodes with peak current densities up to $220 \mathrm{kA} / \mathrm{cm}^{2}$," Applied Physics Letters, vol. 112, p. 103101, 2018.

[9] W. Zhang, S. Watson, J. Figueiredo et al., "Optical direct intensity modulation of a $79 \mathrm{GHz}$ resonant tunneling diodephotodetector oscillator," Optics Express, vol. 27, pp. 1679116797, 2019.

[10] G. Bordiougov and H. Engel, "From trigger to phase waves and back again," Physica D: Nonlinear Phenomena, vol. 215, pp. 25-37, 2006.

[11] S. P. Parsons and J. D. Huizinga, "Phase waves and trigger waves: emergent properties of oscillating and excitable networks in the gut," Journal of Physiology, vol. 596, pp. 48194829, 2018.

[12] K. Narahara, "Dynamics of dissipative solitons developed in a closed traveling-wave field-effect transistor," International Journal of Circuit Theory and Applications, vol. 46, pp. 2000-2010, 2018.

[13] A. A. Abidi, "Phase noise and jitter in CMOS ring oscillators," IEEE Journal of Solid-State Circuits, vol. 41, pp. 1803-1816, 2006.

[14] D. Yardeny, B. A. Kaplan, and Y. Horen, "New structures of four-phase oscillators obtained by strongly interweaving mono-phase limit-cycle oscillators," International Journal of Circuit Theory and Applications, vol. 36, pp. 409-419, 2008.

[15] P. Maffezzoni, B. Bahr, Z. Zhang, and L. Daniel, "Reducing phase noise in multi-phase oscillators," IEEE Transactions on Circuits and Systems I, vol. 63, pp. 379-388, 2016. 
[16] K. Narahara, "Multiphase oscillator using traveling pulses developed in a system of transmission lines with regularly spaced resonant-tunneling diodes," Journal of Infrared, Millimeter and Terahertz Waves, vol. 38, pp. 660-678, 2017.

[17] S. Sawai and K. Narahara, "Submillimeter-wave multiphase oscillation using traveling pulses in a resonant-tunneling diode-oscillator lattice," Journal of Infrared, Millimeter and Terahertz Waves, vol. 42, pp. 426-445, 2021.

[18] W. C. Black and D. A. Hodges, "Time interleaved converter arrays," IEEE Journal of Solid-State Circuits, vol. 15, pp. 1022-1029, 1980.

[19] A. Mazzanti, P. Uggetti, and F. Svelto, "Analysis and design of injection-locked LC dividers for quadrature generation," IEEE Journal of Solid-State Circuits, vol. 39, pp. 1425-1433, 2004.

[20] F. X. Kaertner, "Analysis of white and $\mathrm{f}_{\alpha}$ noise in oscillators," International Journal of Circuit Theory and Applications, vol. 18, pp. 485-519, 1990.

[21] A. Demir, A. Mehrotra, and J. Roychowdhury, "Phase noise in oscillators: a unifying theory and numerical methods for characterization," IEEE Transactions on Circuits and Systems I, vol. 47, pp. 655-674, 2000.

[22] T. Wang and J. Roychowdhury: Rigorous Q factor formulation and characterization for nonlinear oscillators. 2017. 